\title{
A NOTE ON MAPPINGS OF FINITE DISTORTION: EXAMPLES FOR THE SHARP MODULUS OF CONTINUITY
}

\author{
Daniel Campbell and Stanislav Hencl \\ Charles University, Department of Mathematical Analysis \\ Sokolovská 83, 18600 Prague 8, Czech Republic; danuk@centrum.cz \\ Charles University, Department of Mathematical Analysis \\ Sokolovská 83, 18600 Prague 8, Czech Republic; hencl@karlin.mff.cuni.cz
}

\begin{abstract}
We construct a mapping with exponentially integrable distortion which attains a modulus of continuity by Onninen and Zhong, showing that it is sharp.
\end{abstract}

\section{Introduction}

Let $\Omega \subset \mathbf{R}^{n}, n \geq 2$, be a connected and open set. A Sobolev mapping $f \in$ $W_{\text {loc }}^{1,1}\left(\Omega, \mathbf{R}^{n}\right)$ is said to have finite distortion if the Jacobian $J_{f}(x)$, i.e., the determinant of the matrix of derivatives $D f(x)$ is locally integrable and there is a measurable function $K(x) \geq 1$ finite almost everywhere such that

$$
|D f(x)|^{n} \leq K(x) J_{f}(x) \text { a.e. } x \in \Omega .
$$

Here we have used the operator norm of the differential matrix with respect to the Euclidean distance.

If we, moreover, require that $K(x) \in L^{\infty}(\Omega)$, we arrive at mappings of bounded distortion also called quasiregular mappings. In [7] Reshetnyak proved among many other things that quasiregular mappings are Hölder continuous with the exponent $1 / K$, where $K$ is the $L^{\infty}$ norm of the distortion. It has been shown recently that mappings of finite distortion with exponentially integrable distortion

$$
\exp (\lambda K(x)) \in L^{1}(\Omega)
$$

share many nice properties of mappings of bounded distortion. We would like to point the reader's attention to the monographs [1] and [3] for the motivation, applications and the history of the subject.

Our aim is to study the modulus of continuity of the mappings of finite distortion with $\exp (\lambda K) \in L^{1}(\Omega)$. Let us first recall the history of such estimates. First, it was shown by Iwaniec, Koskela and Onninen [2] that mappings in this class are continuous and satisfy

$$
|f(x)-f(y)| \leq \frac{C}{\log ^{1 / n} \log \left(e^{e}+1 /|x-y|\right)}
$$

doi:10.5186/aasfm.2011.3633

2010 Mathematics Subject Classification: Primary 30C65, 46 E35.

Key words: Mapping of finite distortion, modulus of continuity.

The second author was supported by the RSJ algorithmic trading grant. 
This was later improved by Koskela and Onninen [5] to

$$
|f(x)-f(y)| \leq \frac{C}{\log ^{\lambda / n-\varepsilon}(1 /|x-y|)}
$$

and finally using very delicate arguments it has been shown by Onninen and Zhong [6] that

$$
|f(x)-f(y)| \leq \frac{C}{\log ^{\lambda / n}(1 /|x-y|)}
$$

Extremal mappings for continuity of mappings of finite distortion are usually radial maps and therefore the natural candidate for the extremal map is

$$
f_{0}(x)=\frac{x}{|x|} \frac{1}{\log ^{\lambda / n}(1 /|x|)} .
$$

Standard computations (see (2.2) below) give us

$$
K(x)=\frac{n}{\lambda} \log \frac{1}{|x|}
$$

and hence

$$
\int_{B\left(0, \frac{1}{2}\right)} \exp (\lambda K(x)) d x=\int_{B\left(0, \frac{1}{2}\right)} \frac{1}{|x|^{n}} d x=\infty .
$$

This elementary computation suggests that there is some room for improvement in the estimate (1.1) and maybe we can add some supplementary term like $\log \log 1 /|x-y|$ to some negative power to our estimate. We show that, surprisingly, this is not the case and the modulus of continuity (1.1) is already sharp.

Theorem 1.1. Given $\lambda>0$, there is a mapping of finite distortion $f: B\left(0, \frac{1}{2}\right) \rightarrow$ $\mathbf{R}^{n}$ such that

$$
\int_{B\left(0, \frac{1}{2}\right)} \exp (\lambda K(x)) d x<\infty
$$

and

$$
|f(x)-f(0)| \geq \frac{C}{\log ^{\lambda / n}(1 /|x|)} \text { for all } x \in B\left(0, \frac{1}{2}\right) .
$$

There have also been studies on mappings of subexponentially integrable distortion (see e.g. [4]). One requires that

$$
\int_{B} \exp (\mathscr{A}(K(x))) d x<\infty
$$

for some Orlicz function $\mathscr{A}$ and the above mentioned example corresponds to the case $\mathscr{A}(t)=\lambda t$. We call an infinitely differentiable and strictly increasing function $\mathscr{A}:[0, \infty) \rightarrow[0, \infty)$ with $\mathscr{A}(0)=0$ and $\lim _{t \rightarrow \infty} \mathscr{A}(t)=\infty$ an Orlicz function. As usual we impose additional condition

$$
\int_{1}^{\infty} \frac{\mathscr{A}^{\prime}(s)}{s}=\infty .
$$


It is easy to see that the critical functions for this condition are

$$
\begin{aligned}
& \mathscr{A}_{1}(t)=\lambda t, \quad \mathscr{A}_{2}(t)=\lambda \frac{t}{\log (e+t)}, \\
& \mathscr{A}_{3}(t)=\lambda \frac{t}{\log (e+t) \log (e+\log (e+t))} \text { and so on. }
\end{aligned}
$$

We will also require that

(i) $\exists t_{0}>0 \forall t>t_{0} \quad \mathscr{A}^{-1}(n t)<t^{\frac{3}{2}}$,

(ii) $\mathscr{A}^{\prime}(t)$ is non-increasing,

(iii) $b^{\prime}(t)$ is non-increasing for $b(t):=\frac{t}{\mathscr{A}(t)}$,

(iv) $b(0):=\lim _{t \rightarrow 0+} b(t)$ is finite and positive.

Let us note that the critical functions from (1.4) satisfy these conditions and therefore these assumptions are not substantially restrictive. It has been shown in [4] that a mapping $f$ is continuous under the assumptions (1.2) and (1.3) and that the assumption (1.3) is sharp.

It was proved in [6] that under the assumptions (1.2) and (1.3) we have

$$
|f(x)-f(y)| \leq C \exp \left(-\int_{|x-y|}^{R} \frac{d t}{t \mathscr{A}^{-1}(n \log C / t)}\right)
$$

for $|x-y|$ sufficiently small and $B(x, 80 R) \subset \Omega$. Note further that if we put $\mathscr{A}_{1}(t)=\lambda t$ we arrive at the modulus given in (1.1). Our result shows the sharpness of this estimate.

Theorem 1.2. Suppose that an Orlicz function $\mathscr{A}$ satisfies (1.3) and (1.5). Then there is a ball $B:=B(0, r)$ and a mapping of finite distortion $f: B \rightarrow \mathbf{R}^{n}$ such that

$$
\int_{B} \exp (\mathscr{A}(K(x))) d x<\infty
$$

and

$$
|f(x)-f(0)| \geq C \exp \left(-\int_{|x|}^{1 / 2} \frac{d t}{t \mathscr{A}^{-1}(n \log 1 / t)}\right) \text { for all } x \in B
$$

\section{Proofs of the theorems}

To prove Theorem 1.1 we simply set

$$
f(x)=\frac{x}{|x|} \frac{(\log 1 /|x|)^{\frac{a}{\log 1 /|x|}}}{\log ^{\lambda / n}(1 /|x|)},
$$

where $a>0$. The additional term clearly satisfies

$$
\lim _{|x| \rightarrow 0}(\log 1 /|x|)^{\frac{a}{\log 1 /|x|}}=1
$$

and thus the modulus of continuity of our $f$ is exactly as required in (1.1). On the other hand, the additional term slightly affects the distortion and the standard 
computation (see the general case below for details) will give us

$$
K(x) \sim \frac{n}{\lambda} \log \frac{1}{|x|}-\frac{n^{2} a}{\lambda^{2}} \log \log \frac{1}{|x|},
$$

and hence

$$
\int_{B\left(0, \frac{1}{2}\right)} \exp (\lambda K(x)) d x<\infty
$$

for sufficiently large $a$.

To prove Theorem 1.2 , let us put $B:=B\left(0, \min \left\{\exp \left(-t_{0}\right), e^{-e}\right\}\right)$ and choose $\alpha>b(0)^{-1} n^{-2}$. Without loss of generality we can assume that $t_{0}$ is big enough such that

$$
t^{\frac{3}{2}}<\frac{1}{\alpha(\alpha+1)} \frac{t^{2}}{\log t} \text { for all } t>t_{0}
$$

We define the function $f$ as,

$$
f(x):=\frac{x}{|x|} \exp \left(-\int_{|x|}^{\frac{1}{2}} \frac{1}{t \mathscr{A}^{-1}\left(n \log \frac{1}{t}\right)} d t\right)\left(\log |x|^{-1}\right)^{\frac{\alpha+2}{\log |x|^{-1}}} \text { for } x \neq 0 .
$$

Note that

$$
\lim _{|x| \rightarrow 0}\left(\log |x|^{-1}\right)^{\frac{\alpha+2}{\log |x|^{-1}}}=\lim _{|x| \rightarrow 0} \exp \left(\frac{(\alpha+2) \log \log |x|^{-1}}{\log |x|^{-1}}\right)=1,
$$

which easily gives that $f$ satisfies the condition given in (1.6).

Let $\rho:(0, \infty) \rightarrow(0, \infty)$ be a strictly monotone, differentiable function and let us consider the mapping

$$
f(x)=\frac{x}{|x|} \rho(|x|), \quad x \neq 0 .
$$

It can be verified by an elementary computation (see e.g. [1, Chapter 2.6.]) that

$$
\begin{aligned}
|D f(x)| & =\max \left\{\frac{\rho(|x|)}{|x|},\left|\rho^{\prime}(|x|)\right|\right\}, \text { and thus } \\
K(x) & =\max \left\{\frac{\rho(|x|)}{|x|\left|\rho^{\prime}(|x|)\right|}, \frac{|x|\left|\rho^{\prime}(|x|)\right|}{\rho(|x|)}\right\} .
\end{aligned}
$$

It follows that for our mapping we obtain

$$
|D f(x)|=\frac{|f(x)|}{|x|} \max \left\{1,\left(\frac{1}{\mathscr{A}^{-1}\left(n \log |x|^{-1}\right)}+(\alpha+2) \frac{\log \log |x|^{-1}-1}{\log ^{2}|x|^{-1}}\right)\right\} .
$$

Clearly,

$$
\lim _{x \rightarrow 0}\left(\frac{1}{\mathscr{A}^{-1}\left(n \log |x|^{-1}\right)}+(\alpha+2) \frac{\log \log |x|^{-1}-1}{\log ^{2}|x|^{-1}}\right)=0
$$

and therefore the greater element is the first one. From (1.5) (i) and (2.1) we obtain

$$
\mathscr{A}^{-1}(n t)<t^{\frac{3}{2}}<\frac{1}{\alpha(\alpha+1)} \frac{t^{2}}{\log t} \text { for all } t>t_{0} .
$$

This, however, implies that

$$
\alpha(\alpha+1) \frac{\mathscr{A}^{-1}(n t) \log t}{t^{2}}<1 \text { for all } t>t_{0} .
$$


Now, by multiplying both sides by $\frac{\mathscr{A}^{-1}(n t) \log t}{t^{2}}$ and by substituting $t=\log \left|x^{-1}\right|$ we get that

$$
\mathscr{A}^{-1}\left(n \log |x|^{-1}\right) \frac{\log \log |x|^{-1}}{\log ^{2}|x|^{-1}}>\alpha(\alpha+1)\left(\mathscr{A}^{-1}\left(n \log |x|^{-1}\right) \frac{\log \log |x|^{-1}}{\log ^{2}|x|^{-1}}\right)^{2}
$$

for all $x \in B$. Using this fact and because $\log \log |x|^{-1}>1$ for all $x \in B$, we deduce that

$$
\begin{aligned}
K(x) & =\frac{1}{\left(\frac{1}{\mathscr{A}^{-1}\left(n \log |x|^{-1}\right)}+(\alpha+2) \frac{\log \log |x|^{-1}-1}{\log ^{2}|x|^{-1}}\right)} \\
& \leq \frac{\mathscr{A}^{-1}\left(n \log |x|^{-1}\right)}{1+(\alpha+1) \mathscr{A}^{-1}\left(n \log |x|^{-1}\right) \frac{\log \log |x|^{-1}}{\log ^{2}|x|^{-1}}} \\
& \leq \mathscr{A}^{-1}\left(n \log |x|^{-1}\right)\left(1-\alpha \mathscr{A}^{-1}\left(n \log |x|^{-1}\right) \frac{\log \log |x|^{-1}}{\log ^{2}|x|^{-1}}\right)=: \tilde{K}(x) .
\end{aligned}
$$

Note that

$$
\mathscr{A}^{-1}\left(n \log |x|^{-1}\right)-\tilde{K}(x)=\alpha n^{2}\left(\frac{\mathscr{A}^{-1}\left(n \log |x|^{-1}\right)}{n \log |x|^{-1}}\right)^{2} \log \log |x|^{-1} .
$$

By (1.5) (iii) we obtain that

$$
b(s)-b(0)=b^{\prime}(\xi) s \geq b^{\prime}(s) s
$$

and therefore

$$
\mathscr{A}^{\prime}(s)\left(\frac{s}{\mathscr{A}(s)}\right)^{2}=\frac{b(s)-s b^{\prime}(s)}{b^{2}(s)} b^{2}(s) \geq b(0) .
$$

From (1.5) (ii) we know that $\mathscr{A}^{\prime}(t)$ is a non-increasing function and hence

$$
\mathscr{A}(a-d)=\mathscr{A}(a)-\mathscr{A}^{\prime}(\xi) d \leq \mathscr{A}(a)-\mathscr{A}^{\prime}(a) d
$$

for some $\xi \in(a-d, a)$. We now use (2.5) putting

$$
a:=\mathscr{A}^{-1}\left(n \log \frac{1}{|x|}\right), \quad d:=\mathscr{A}^{-1}\left(n \log \frac{1}{|x|}\right)-\tilde{K}(x)
$$

using (2.3) and then (2.4) (where we put $s:=\mathscr{A}^{-1}\left(n \log |x|^{-1}\right)$ ) to get that

$$
\begin{aligned}
& \mathscr{A}(K(x)) \leq \mathscr{A}(\tilde{K}(x)) \\
& \leq \mathscr{A}\left(\mathscr{A}^{-1}\left(n \log |x|^{-1}\right)\right)-\mathscr{A}^{\prime}\left(\mathscr{A}^{-1}\left(n \log |x|^{-1}\right)\right)\left[\mathscr{A}^{-1}\left(n \log |x|^{-1}\right)-\tilde{K}(x)\right] \\
& \leq n \log |x|^{-1}-\alpha n^{2} \mathscr{A}^{\prime}\left(\mathscr{A}^{-1}\left(n \log |x|^{-1}\right)\right)\left(\frac{\mathscr{A}^{-1}\left(n \log |x|^{-1}\right)}{n \log |x|^{-1}}\right)^{2} \log \log |x|^{-1} \\
& \leq n \log |x|^{-1}-b(0) \alpha n^{2} \log \log |x|^{-1} .
\end{aligned}
$$

But this, for $\alpha>b(0)^{-1} n^{-2}$, yields

$$
\begin{aligned}
\int_{B} \exp (\mathscr{A}(K(x))) d x & \leq \int_{B} \exp \left(n \log \frac{1}{|x|}-b(0) \alpha n^{2} \log \log \frac{1}{|x|}\right) d x \\
& \leq \int_{B} \frac{1}{|x|^{n} \log ^{b(0) \alpha n^{2}} \frac{1}{|x|}} d x<\infty .
\end{aligned}
$$




\section{References}

[1] Astala, K., T. Iwaniec, and G. Martin: Elliptic partial differential equations and quasiconformal mappings in the plane. - Princeton Math. Ser. 48, Princeton Univ. Press, Princeton, NJ, 2009.

[2] Iwaniec, T., P. Koskela, and J. Onninen: Mappings of finite distortion: Monotonicity and continuity. - Invent. Math. 144, 2001, 507-531.

[3] Iwaniec, T., and G. Martin: Geometric function theory and nonlinear analysis. - Oxford Math. Monogr., Clarendon Press, Oxford, 2001.

[4] Kauhanen, J., P. Koskela, J. Malý, J. Onninen, and X. Zhong: Mappings of finite distortion: Sharp Orlicz-conditions. - Rev. Mat. Iberoamericana 19, 857-872, 2003.

[5] Koskela, P., and J. Onninen: Mappings of finite distortion: the sharp modulus of continuity. - Trans. Amer. Math. Soc. 355:5, 2003, 1905-1920.

[6] Onninen, J., and X. ZhOnG: A note on mappings of finite distortion: the sharp modulus of continuity. - Michigan Math. J. 53:2, 2005, 329-335.

[7] Reshetnyak, Yu. G.: Space mappings with bounded distortion. - Transl. Math. Monogr. 73, Amer. Math. Soc., 1989.

Received 28 May 2010 